\title{
Is GSK3 $\beta$ a molecular target of chloroquine treatment against COVID-19?
}

\author{
Mohammed Noor Embi ${ }^{*}$, Nagesswary Ganesan, Hasidah Mohd Sidek \\ Department of Biological Sciences and Biotechnology, Faculty of Science and Technology, Universiti Kebangsaan Malaysia, Selangor, Malaysia.
}

SUMMARY The recent clinical trial reports pertaining to the efficacy of chloroquine and hydroxychloroquine against COVID-19 albeit yet to be validated with larger clinical trials, have sparked much interest globally to evaluate whether this anti-malarial drug can be repurposed for the treatment of COVID-19. In addition to its anti-viral activity, the anti-inflammatory activity of chloroquine may also contribute to its efficacy. Based on our data obtained from an animal infection model of melioidosis (a disease caused by the bacteria Burkholderia pseudomallei), treatment with chloroquine can result in the phosphorylation and consequent inhibition of glycogen synthase kinase-3 $\beta$ (GSK3 $\beta$ ). This serine/threonine protein kinase is now recognised as a point of convergence for host inflammatory response. In view of this, it is plausible that the mechanism for the anti-inflammatory effect of chloroquine against COVID-19 involves inhibition of host GSK3 $\beta$.

Keywords Chloroquine, COVID-19, GSK3 $\beta$, anti-inflammatory

The molecular basis by which chloroquine dampens the host overwhelming inflammatory response (cytokine storm) during infection is still not fully understood. As in malaria, pathogenesis in viral infection may also be related to dysfunction in the regulation of pertinent signalling pathways; for example aberrant GSK3 $\beta$ signalling. Our notion is based on the understanding that GSK $3 \beta$ is a molecular hub linking numerous signalling pathways in the cell, including host-directed inflammatory response. Lithium chloride $(\mathrm{LiCl})$, a wellknown GSK3 inhibitor, has been reported to suppress avian coronavirus infectious bronchitis where the antiviral activity of lithium was attributed to its cellular effect (1). Most recently, Nowak \& Walkowiak (2020) (2) proposed $\mathrm{LiCl}$ to be further explored as a potential therapeutic for the treatment of COVID-19. The reported efficacy of chloroquine in recent clinical trials to treat COVID-19 $(3,4)$ may also be attributed to a mechanism of action that involve inhibition of GSK $3 \beta$. In our laboratory, we have shown that chloroquine treatment in experimental animal melioidosis modulated cytokine levels and increased animal survivability via inhibition of GSK3 $\beta$ (5). Our analysis revealed that chloroquine resulted in phosphorylation and consequent inhibition of GSK $3 \beta$ in the liver. In a subsequent study ( 6 ), we concluded that chloroquine is a plausible candidate for repurposing in the treatment of melioidosis. It is possible that the mechanism for the anti-viral activity of chloroquine, specifically its anti-inflammatory effect involves inhibition of GSK3 $\beta$ in lung epithelial and immune cells. The lead author's 1980 publication (7) first identified GSK3 forty years ago. This kinase, initially described as a key enzyme involved in glycogen metabolism, is now known to regulate a wide array of cellular processes. Dysregulation of this kinase is implicated in several diseases including bipolar disorder, diabetes mellitus, Alzheimer's disease, inflammation, and cancer $(8,9)$. Further research to better understand the molecular basis of the anti-viral effects of chloroquine can have far-reaching clinical implications.

\section{References}

1. Harrison SM, Tarpey I, Rothwell L, Kaiser P, Hiscox JA. Lithium chloride inhibits the coronavirus infectious bronchitis virus in cell culture. Avian Pathol. 2007; 36:109-114.

2. Nowak JK, Walkowiak J. Is lithium a potential treatment for the novel Wuhan (2019-nCoV) coronavirus? A scoping review. F1000Research. 2020; 9:93.

3. Gao J, Tian Z, Yang X. Breakthrough: Chloroquine phosphate has shown apparent efficacy in treatment of COVID-19 associated pneumonia in clinical studies. Biosci Trends. 2020; 14:72-73.

4. Gautret $\mathrm{P}$, Lagierac JC, Parola $\mathrm{P}$, et al. Hydroxychloroquine and azithromycin as a treatment of COVID-19: results 
of an open-label non-randomized clinical trial. Int J Antimicrob Agents. 2020;105949. doi: 10.1016/ j.ijantimicag.2020.105949.

5. Ganesan N, Embi N, Hasidah M. The anti-malarial chloroquine modulated cytokine levels and increased animal survivability via Akt-mediated inhibition of GSK3 $\beta$ in Burkholderia pseudomallei-infected mice. Trop Biomed. 2018; 35:709-723.

6. Ganesan N, Embi N, Hasidah MS. Potential of repurposing chloroquine as an adjunct therapy for melioidosis based on a murine model of Burkholderia pseudomallei infection. Trop Biomed. 2020.

7. Embi N, Rylatt DB, Cohen P. Glycogen synthase kinase-3 from rabbit skeletal muscle. Separation from cyclic-AMPdependent protein kinase and phosphorylase kinase. Eur J Biochem. 1980; 107:519-527.

8. Beurel E, Grieco SF, Jope RS. Glycogen synthase kinase-3 (GSK3): regulation, actions, and diseases.
Pharmacol Ther. 2015; 148:114-131.

9. Xu M, Wang S, Zhu L, Wu P, Dai W, Rakesh K. Structure-activity relationship (SAR) studies of synthetic glycogen synthase kinase- $3 \beta$ inhibitors: a critical review. Eur J Med Chem. 2019; 164:448-470.

Received March 31, 2020; Revised April 17, 2020; Accepted April 18, 2020.

*Address correspondence to:

Mohammed Noor Embi, Department of Biological Sciences and Biotechnology, Faculty of Science and Technology, Universiti Kebangsaan Malaysia, 43600 UKM Bangi, Selangor, Malaysia.

E-mail: noormb@ukm.edu.my

Released online in J-STAGE as advance publication April 21, 2020. 